\title{
Is BRCA mutational status a predictor of platinum-based chemotherapy related hematologic toxicity in high-grade serous ovarian cancer patients?
}

Federica Tomao, Lucia Musacchio, Federica Di Mauro, Serena Maria Boccia, Violante Di Donato, Antonella Giancotti, Giorgia Perniola, Innocenza Palaia, Ludovico Muzii, Pierluigi Benedetti Panici

Department of Maternal and Child Health and Urological Sciences, University "Sapienza", Policlinico "Umberto I" Rome, Italy

\section{Objective}

To evaluate hematologic adverse effect profiles associated with frontline platinum-based chemotherapy in ovarian cancer patients according to BRCA $1 / 2$ mutational status.

\section{Methods}

Patients with high-grade serous ovarian cancer and a known BRCA mutational status who received in frontline 6 cycles of Carboplatin (AUC 5) plus Paclitaxel $175 \mathrm{mg} / \mathrm{mq}$ were retrospectively selected from our databases. Hematologic toxicity profiles of BRCA mutated patients were compared to nonmutated patients, according to EORTC Common Terminology Criteria for Adverse Events (CTCAE_4.02)

\section{Results}

Totally, 176 women of whom 58 (33\%) were BRCA1/2 mutation carriers - 40 BRCA1 (69\%) and 18 (31\%) BRCA2 mutations carriers - and 118 (67\%) non-carriers were identified. A significant higher frequency of thrombocytopenia (24\% vs $5 \%$; p b 0.001), anemia (21\% vs 7\%; $p=0.006)$ and neutropenia $(62 \%$ vs $27 \% ; p \leq 0.001)$ was observed in BRCA mutated patients, resulting in a higher percentage of granulocytecolony stimulating growth factors injection ( $12 \%$ versus $1 \%, \mathrm{p} \mathrm{b}$ $0.001)$ and dose delay $(19 \%$ versus $27 \%, p=0.005)$. The multivariate analysis confirmed that granulocyte-colony stimulating growth factors injection and dose delay were statistically significantly more frequent in BRCA mutated patients (OR 2.567, 95\% Cl 1.136-5.798, $p=0.035$; OR 3.860, $95 \% \mathrm{Cl} 1.098-13.570, p=0.023)$. Finally, the total number of hematologic adverse events compared between the two groups of patients during the entire treatment period showed a substantial higher rate of hematologic adverse events in BRCA mutated population.

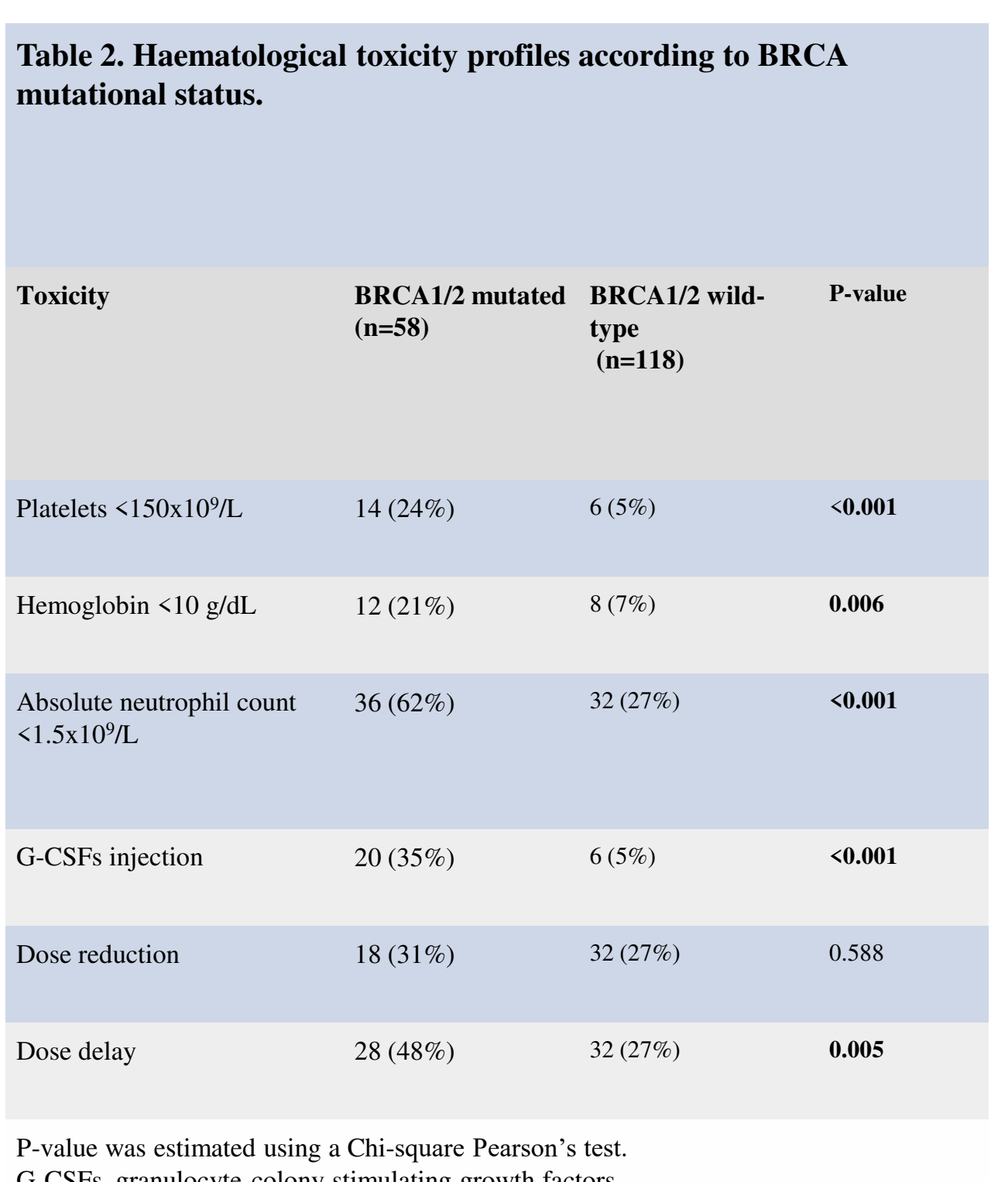
G-CSFs, granulocyte-colony stimulating growth factors.

Figures show rates of anemia and neutropenia in NACT patients per cycle, according to BRCA mutational status
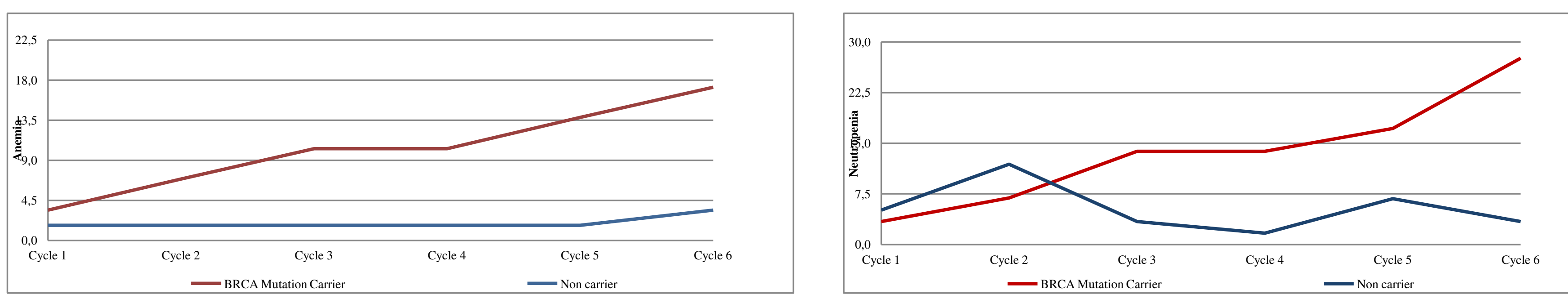

\section{Conclusions:}

Germline BRCA $1 / 2$ mutations are associated with a higher hematologic toxicity in patients with ovarian cancer who underwent platinum-based chemotherapy. 